\title{
Candida glabrata epithelial cell damage
}

Marina Pekmezovic', Selene Mogavero' and Bernhard Hube ${ }^{1,2}$

${ }^{1}$ Department of Microbial Pathogenicity Mechanisms, Hans-Knöll-Institute, Jena, Germany;

2 Friedrich-Schiller- University Jena, Germany

\section{Introduction}

Candida glabrata is the second most common pathogenic Candida species of humans. It is normally found among the resident microbiota of healthy individuals' mucosae, but can cause disease when host barriers or immunity are impaired. Disease states range from superficial skin and mucosal infections to systemic infections. The transition from commensalism to pathogenicity is well described in $C$. albicans and it occurs in three major steps: adhesion, invasion and damage of host cells. In C. glabrata, on the other hand, he same transition is still poorly elucidated. Since $C$. glabrata is phylogenetically very different from $C$. albicans and lacks some of the major $C$. albicans virulence attributes, such as ability to form hyphae, it is unlikely that the steps leading to epithelial damage have common mechanisms between these two very different fungi.

\section{Aim of the study}

We aimed to investigate the adhesion, invasion and damage potential of C. glabrata during infection of vaginal human epithelial cells (ECs) in vitro.
In vitro vaginal epithelial cell model

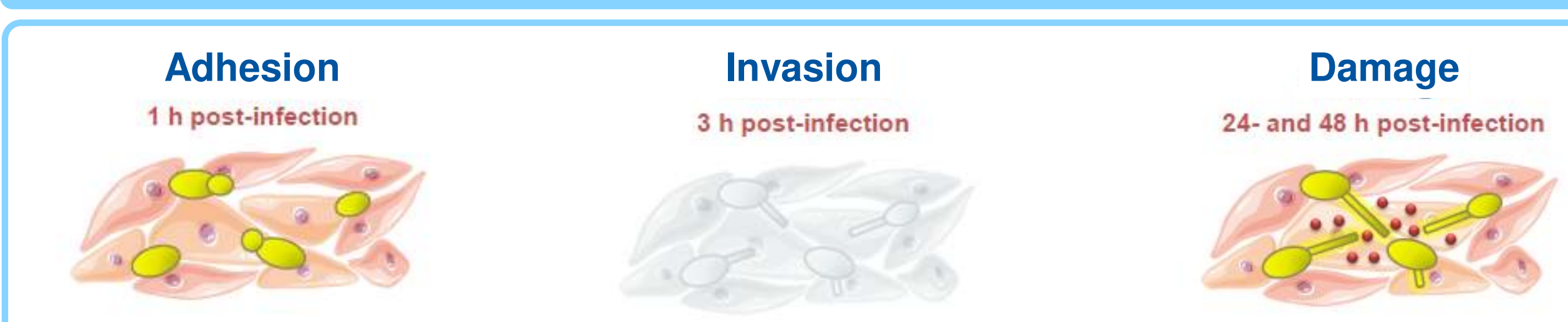

10-15\% of adhesion
Significant damage $48 \mathrm{~h}$ post-infection
Mutant library screening for epithelial damage

C. glabrata deletion mutant library screening

620 strains (Schwarzmüller et al. PLoS Pathog. 2014)
Overnight cultures in YPD
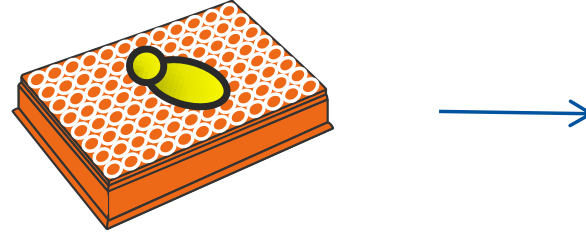

Infection of vaginal ECs A431 in RPMI

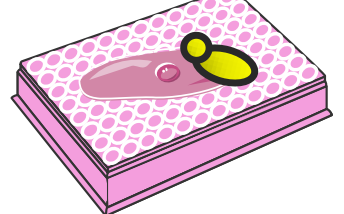

Damage measurement
- Damage was determined by measuring the release of lactate dehydrogenase (LDH)

- Comparisons were made with C. glabrata wildtype (WT: ATCC2001 $\Delta$ trp1,

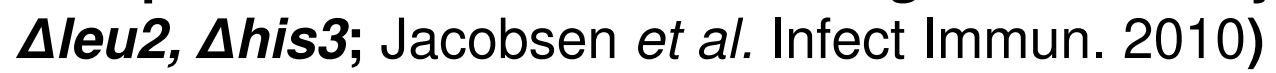

\section{Conclusions}

\section{Adherence to host cells does not lead to invasion but it could activate host responses that ultimately cause epithelial damage.}

These data suggest a hyphal independent damage mechanism of C. glabrata, which requires adhesion, but not invasion. In further studies, we aim at characterizing the role of damage associated genes and elucidating the processes leading to damage.
Mutant selection based on damage

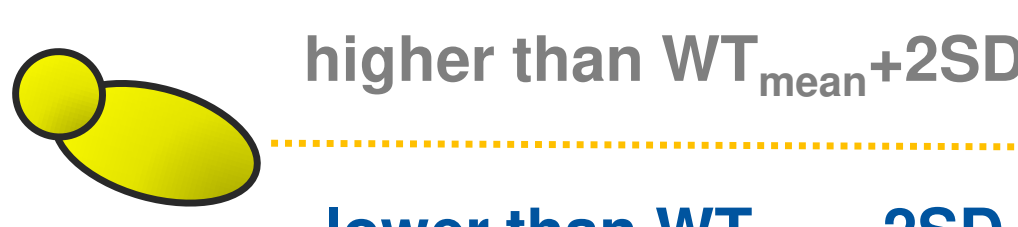

lower than $\mathrm{WT}_{\text {mean }}-\mathbf{2 S D}$

$\downarrow$
Low-damaging (LD) mutants
$\mathrm{n}=40$

Gene ontology (GO)-term analysis:

$32.5 \%(13 / 40)$ genes involved in adhesion and cell wall organization $(p<0.01)$

These mutants showed the reduction in both damage and adhesion

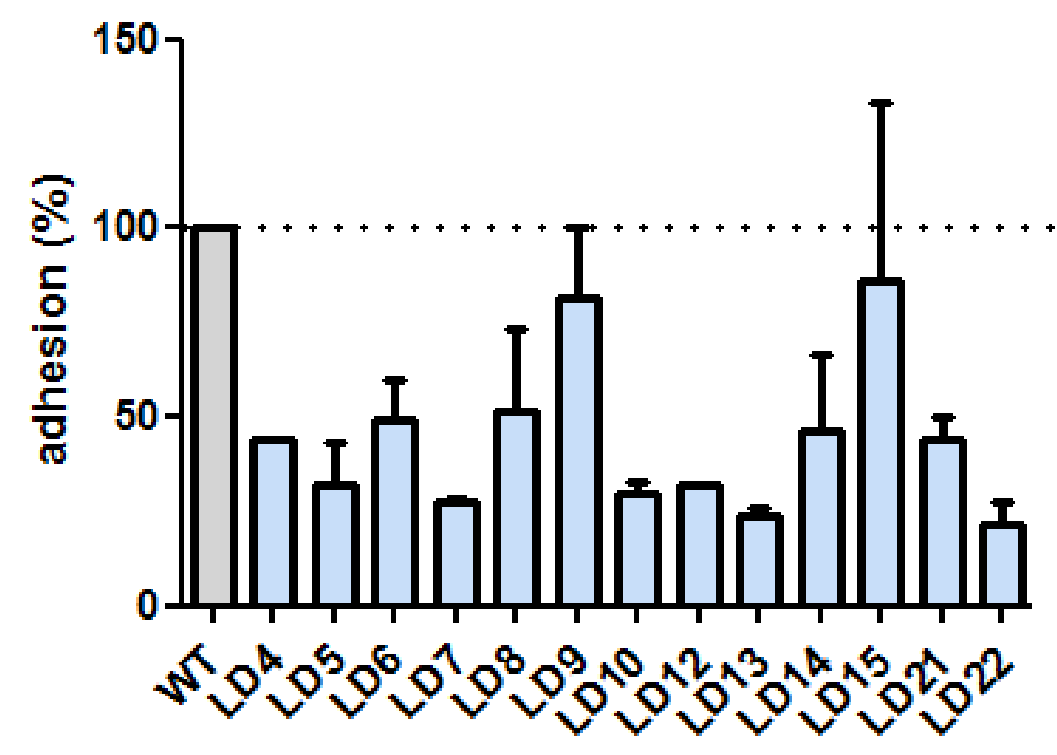

